\title{
Real time wavefront control system for the Large Synoptic Survey Telescope (LSST)
}

George Z. Angeli, Bo Xin, Charles Claver, Douglas MacMartin, Douglas Neill, et al.

George Z. Angeli, Bo Xin, Charles Claver, Douglas MacMartin, Douglas Neill, Matthew Britton, Jacques Sebag, Srinivasan Chandrasekharan, "Real time wavefront control system for the Large Synoptic Survey Telescope (LSST)," Proc. SPIE 9150, Modeling, Systems Engineering, and Project Management for Astronomy VI, 91500H (4 August 2014); doi: $10.1117 / 12.2055390$

Event: SPIE Astronomical Telescopes + Instrumentation, 2014, Montréal, Quebec, Canada 


\title{
Real Time Wavefront Control System for the Large Synoptic Survey Telescope (LSST)
}

\author{
George Z. Angeli*a ${ }^{\mathrm{a}}$, Bo Xin ${ }^{\mathrm{a}}$, Charles Claver ${ }^{\mathrm{a}}$, Douglas MacMartin ${ }^{\mathrm{b}}$, Douglas Neill ${ }^{\mathrm{a}}$, Matthew \\ Britton $^{\mathrm{c}}$, Jacques Sebag ${ }^{\mathrm{a}}$, and Srinivasan Chandrasekharan ${ }^{\mathrm{a}}$ \\ ${ }^{a}$ Large Synoptic Survey Telescope, 950 N. Cherry Ave., Tucson, AZ, 85719 USA \\ ${ }^{\mathrm{b}}$ California Institute of Technology, 1200 E. California Blvd., Pasadena, CA, 91125 USA \\ ${ }^{\mathrm{c}}$ the Optical Sciences Company, 1341 S. Sunkist St., Anaheim, CA, 92806 USA
}

\begin{abstract}
The LSST is an integrated, ground based survey system designed to conduct a decade-long time domain survey of the optical sky. It consists of an 8-meter class wide-field telescope, a 3.2 Gpixel camera, and an automated data processing system. In order to realize the scientific potential of the LSST, its optical system has to provide excellent and consistent image quality across the entire 3.5 degree Field of View. The purpose of the Active Optics System (AOS) is to optimize the image quality by controlling the surface figures of the telescope mirrors and maintaining the relative positions of the optical elements. The basic challenge of the wavefront sensor feedback loop for an LSST type 3-mirror telescope is the near degeneracy of the influence function linking optical degrees of freedom to the measured wavefront errors. Our approach to mitigate this problem is modal control, where a limited number of modes (combinations of optical degrees of freedom) are operated at the sampling rate of the wavefront sensing, while the control bandwidth for the barely observable modes is significantly lower. The paper presents a control strategy based on linear approximations to the system, and the verification of this strategy against system requirements by simulations using more complete, non-linear models for LSST optics and the curvature wavefront sensors.
\end{abstract}

Keywords: Wavefront control, image quality, active optics, LSST

\section{INTRODUCTION}

The LSST is an integrated, ground based survey system designed to conduct a decade-long time domain survey of the optical sky. It consists of an 8-meter class wide-field telescope, a 3.2 Gpixel camera, and an automated data processing system. The LSST will enable a wide variety of simultaneous, complementary science investigations, from searches for small, near-Earth bodies to precision astrometry, from systematic monitoring for transient phenomena to understanding the nature of dark energy and dark matter.

In order to realize the scientific potential of the LSST, its optical system has to provide excellent and consistent image quality across the entire 3.5 degree Field of View (FoV). The purpose of the Active Optics System (AOS) is to optimize the image quality by controlling the surface figures of the telescope mirrors and maintaining the relative positions of the three optical systems: the monolith glass substrate of the Primary and Tertiary Mirrors (M1M3), the Secondary Mirror (M2), and the Camera. Although open loop (feed-forward) set points of this control are provided by a Look-Up-Table, the delivered image is further optimized by utilizing continuous, real time wavefront measurements. This paper focuses on the feedback loop based on these wavefront measurements.

The detailed overview of the entire LSST AOS, including its hardware components and operations is provided elsewhere [1].

The LSST optical systems is shown in Figure 1. The basic challenge of the wavefront sensor feedback loop for this 3mirror telescope is the near-degeneracy of the influence function linking optical degrees of freedom to the measured wavefront errors. In other words, the reconstruction of some combinations of misalignments and mirror deformations from wavefront information is ambiguous, especially in the presence of large atmospheric background aberrations.

*gangeli@1sst.org; phone 1520 318-8413; fax 1520 318-8590

Modeling, Systems Engineering, and Project Management for Astronomy $\mathrm{VI}$, edited by George Z. Angeli, Philippe Dierickx. Proc. of SPIE Vol. 9150, 91500H

(C) 2014 SPIE $\cdot$ CCC code: $0277-786$ X/14/\$18 $\cdot$ doi: $10.1117 / 12.2055390$

Proc. of SPIE Vol. $915091500 \mathrm{H}-1$ 


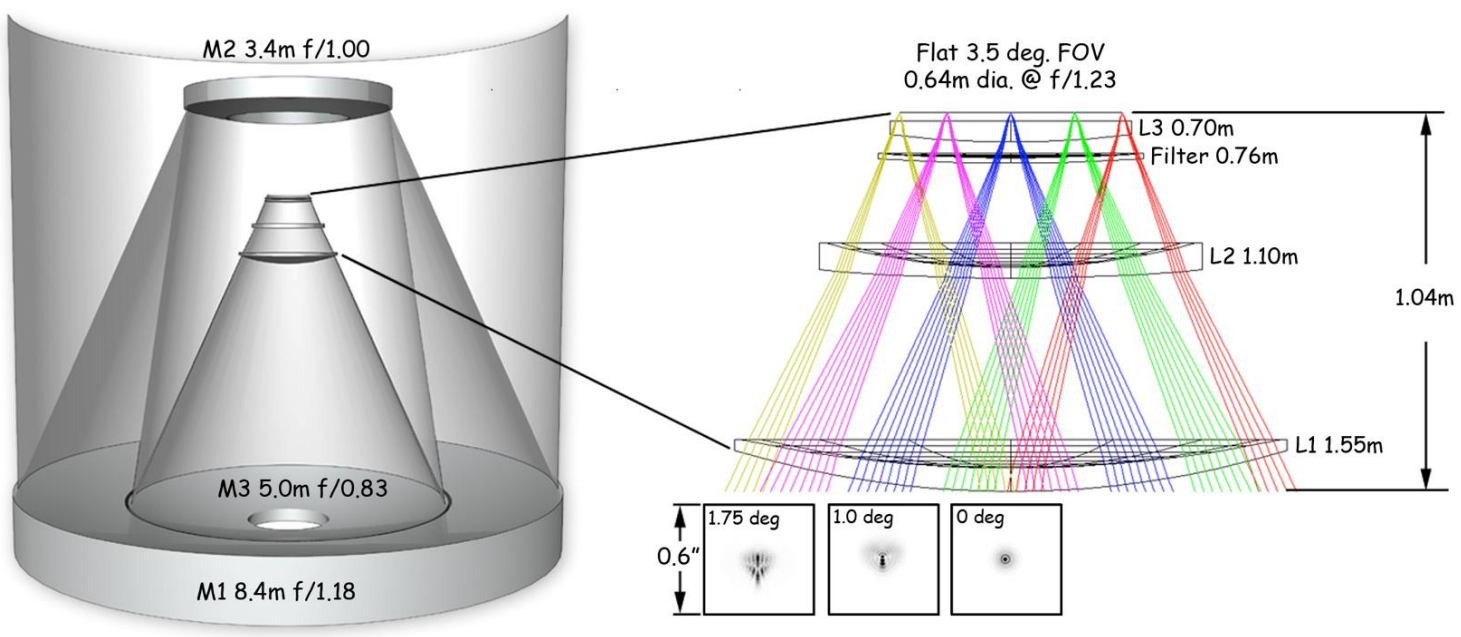

Figure 1 The LSST optical system consists of a modified Paul-Baker 3-mirror telescope and a camera with refractive components. As the insert indicates, design image quality is consistently excellent across the entire field of view.

Our approach to mitigate this problem is modal control, combined with a control law optimized for simultaneously reducing wavefront errors and actuator response. A limited number of modes (combinations of optical degrees of freedom) are operated at the full sampling rate of the wavefront sensing, while the control bandwidth for the barely observable modes is significantly lower.

Compared to most other telescopes, the LSST uses a relatively rapid observing cadence. The LSST survey is conducted through a series of "visits" in order to cover the sky that is currently visible. Each visit consists of a pair of 15-second exposures taken back-to-back. Each exposure requires an elapsed time of 16 seconds to allow for the 1-second transit time of the shutter blades. CCD readout takes 2 seconds, where the second exposure readout is done in parallel with repointing the telescope to the next visit. A typical visit lasts for $\sim 39$ seconds -34 seconds for the two exposures and one image readout followed by 5 seconds to reposition the telescope to the next adjacent field and the final image readout.

The LSST is utilizing 4 curvature wavefront sensors (WCS) at the four corner of its field of view. The actual geometry is described in [1]. As the WCSs are behind the science shutter, their exposure time $(2 \times 15 s)$ and sampling rate $(\sim 39 \mathrm{~s})$ are tied to the timing of the science visits.

An early proposal for LSST wavefront correction was published by Phillion et al. [2]. It was based on a slightly different focal plane geometry and utilized 16 curvature wavefront sensors evenly distributed across the field of view, albeit not all of them were used in the various simulations. A major conclusion of the paper was that 4 wavefront sensors evenly distributed on the circumference of the field can provide suitable information for wavefront control. Manuel et al. [3] investigated the performance of 4 curvature wavefront sensors in the LSST baseline configuration, and concluded that a sufficient number of reference stars are statistically available for any LSST wavelength band and zenith angle. A consequent iteration of the feedback algorithm was reported by Liang et al. [4]. The simulations did not yet included proper atmospheric noise propagation and the feedback was optimized for wavefront error, not FWHM.

The near degeneracy of three-mirror telescope optical response was pointed out by Schechter and Levinson [5]. Their paper is also a good summary of the analytical approach to derive and analyze the optical response of an LSST-like telescope.

LSST being a very large Field-of-View telescope, the image quality may vary across the full field by non-negligible amounts. Consequently, the relevant performance metric is the mean FWHM over the entire focal plane. The overall image quality specification allocated to the Telescope, Camera, and the inherent design aberration of the wide-field telescope are specified in the Observatory System Specification. Further subsystem allocations can be found in the Telescope and Camera error budgets.

The error budget reflects the state of the system after all the corrections possible have been applied, including the optical wavefront sensor feedback. From a controls point of view, the errors can be grouped into 7 major categories. 
1. Inherent design aberrations of the optical system are very low; they contribute 80 mas to the error budget. These aberrations are included in our analysis not because the wavefront control system can alter them, but because they cannot be excluded from the full nonlinear simulations.

2. Telescope errors constant in time are corrected by the Look-Up-Table containing the best possible set of actuator values. The residuals of these errors, after LUT compensation are captured in the error budget: (i) mirror and lens surface fabrication errors, (ii) other manufacturing and installation errors, including the focal plane, (iii) mirror coating thickness non-uniformity, and (iv) fabricated relative positioning errors of M1 and M3. Fitting errors, i.e. the discrepancy between the actual effects and the ability of the realized actuator geometry to correct them, is included in these residuals. While the precision of the LUT based corrections depend on the capabilities of the wavefront sensors, as they provide the final refinement to the LUT, these errors will be estimated separately from the optimization of the wavefront control loop.

3. Telescope errors that are changing faster than the temporal bandwidth of the wavefront control loop cannot be corrected properly by this loop. These errors include: (i) wind buffeting, (ii) tracking/guiding jitter, (iii) vibration, and (iv) dome seeing. The guider system and corresponding error residuals are investigated in a separate study.

4. Wavefront correction errors are introduced by the wavefront control system; these errors do not exist in the system without the wavefront sensors feedback loop. They include: (i) atmospheric residuals filtered through the control loop, (ii) wavefront sensor noise in estimating the actual wavefront from measurements, (iii) uncertainty in reconstructing the telescope state from the wavefront estimates, and (iv) control time lag accounting for the relatively long sampling time. The overall contribution of the optical feedback to the error budget is 79 mas.

5. Slowly varying errors are mostly corrected by the wavefront control loop. More precisely, the components of these errors falling into the temporal and spatial frequency bands of the loop are corrected. The categories of these errors are thermally and gravitationally induced rigid body position shifts and low spatial order surface errors for each optical element. Since the LUT will continuously be updated based on wavefront sensor measurements, eventually only the uncertainty in these slowly changing errors will remain for the loop to correct. However, this study demonstrates that the required image quality can be reached from the initial alignment of the telescope achieved by other means (FEA, optics shop tests, and laser tracker alignment). The telescope residual shape and position errors due to gravitational and thermal effects contribute to the image size of 152 mas at the highest probability elevation angle of $46^{\circ}$.

6. Camera aberrations are a special type of slowly varying errors, as they are not actively corrected. However, the wavefront control loop still partially compensates for these errors. Their contribution is 69 mas to the overall error budget.

7. Camera errors constant in time are not corrected during operations, although the wavefront control loop compensates for some of these errors. The most prominent ones in this category are: (i) initial position alignment errors and (ii) optical fabrication errors. Their combined contribution to the error budget is 80 mas.

The basic concern of this paper is the balance between the last four error categories. The optical feedback loop is working properly if it can reduce the slowly varying errors to the level required by the error budget, without introducing excessive additional image degradation.

\section{ENVIRONMENTAL CONSTRAINTS AND PERTURBATIONS}

The LSST AOS is driven primarily by perturbations due to the observing environment - mainly the temperature of various components and its gradients - and the position of the telescope - mainly the elevation angle of the observation. Further, our ability to extract the necessary optical measurements during observations is limited by the quality of the atmosphere above the observatory site and thermal seeing conditions in and around the telescope.

\subsection{Atmospheric seeing}

The LSST observatory will be constructed on Cerro Pachón, Chile on the peak known as Cerro Peñón. An active monitoring effort was maintained on the summit to measure seeing and temperature profiles of the boundary layer. 
Gemini South ( $\sim 1 \mathrm{~km}$ away from the LSST site) also maintains ongoing seeing and turbulence profile measurements using a MASS/DIMM instrument as part of routine operations. The distribution of atmospheric seeing from DIMM measurements indicate that El Peñón is very similar in characteristics to the Gemini and SOAR sites also along the Cerro Pachón ridge. The equivalent $500 \mathrm{~nm} r_{0}$ (Fried parameter) for the $25^{\text {th }}$, median, and 75 th quartiles are $9.5 \mathrm{~cm}, 12 \mathrm{~cm}$, and $15 \mathrm{~cm}$, respectively.
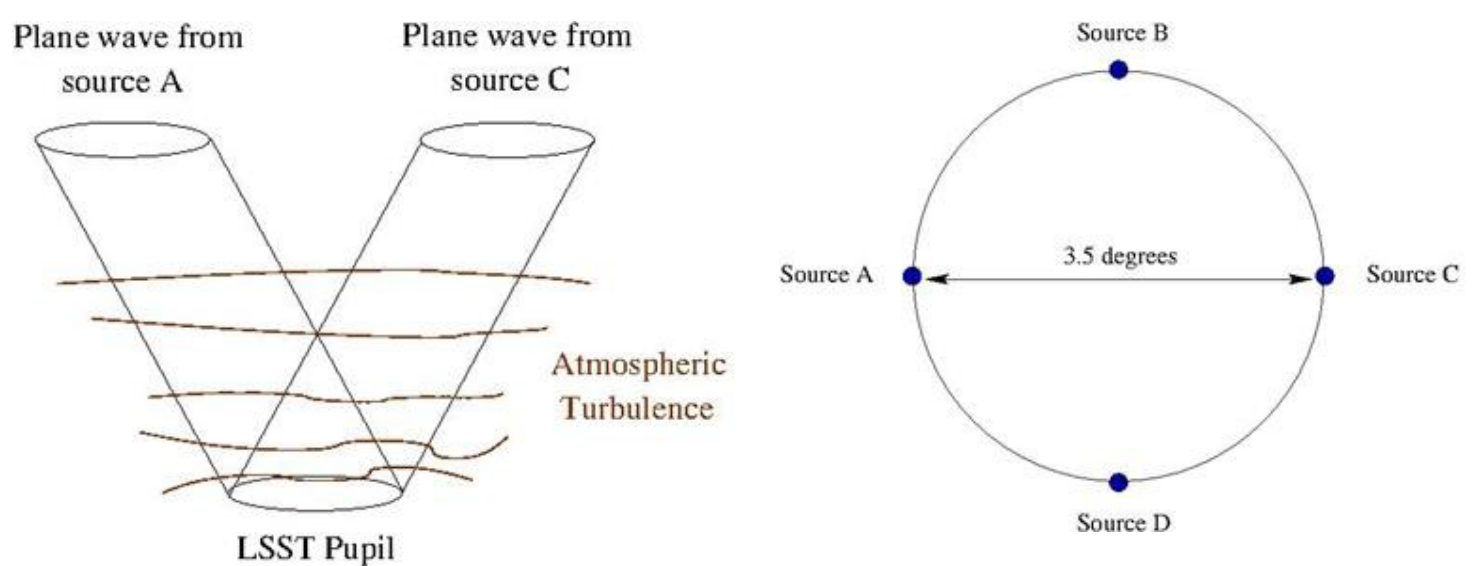

Figure 2 Wavefronts were propagated from each of the 4 sources corresponding to the 4 WCS through the phase screens of the atmosphere and to the LSST entrance pupil and then to the far field, yielding the short exposure pupil plane wavefront and focal plane point spread function at reference wavelength $\lambda=770 \mathrm{~nm}$.

The effect of an outer scale reduces the delivered image size from finite large apertures. The estimated 500nm median delivered image quality $(F W H M)$ from LSST at El Peñón with an outer scale of $20 \mathrm{~m}$ is 0.65 arcsec. Scaled to the centers of the $\mathrm{r}(624 \mathrm{~nm})$ and $\mathrm{i}(770 \mathrm{~nm})$ band filters, it becomes 0.62 and 0.60 arcsec, respectively. For this reason the LSST Science Requirements document defines the allowed range of overall image quality for a fiducial atmosphere delivering $0.6 \operatorname{arcsec} F W H M$.

For the purpose of analyzing and simulating the performance of the LSST AOS we have used atmosphere models generated by the Arroyo software library [6] using a 6-layer model of the vertical turbulence structure. The layer weights were chosen to match the mean vertical profile for Cerro Pachon. These models do not include an outer scale, therefore to approximate the expected residual atmospheric phase errors we have chosen an $r_{0}=17 \mathrm{~cm}$, which is equivalent to the SRD fiducial atmosphere at our simulation wavelength of $770 \mathrm{~nm}$.

Phase screens were generated with $0.065 \mathrm{~m}$ sampling using standard numerical techniques and wind vectors were drawn from a Bufton model. Wavefronts were propagated from each of the four sources through the phase screens and to the LSST entrance pupil and then to the far field, yielding the short exposure pupil plane wavefront and point spread function at reference wavelength (Figure 2).
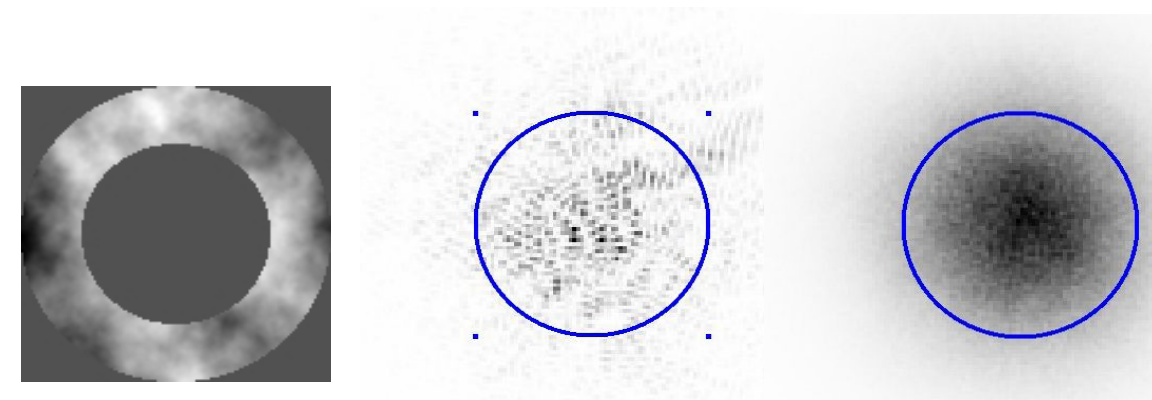

Figure 3 At left is shown a single short exposure $(50 \mathrm{~ms})$ wavefront phase degradation arising solely from atmospheric turbulence. At center is shown a corresponding, single short exposure "speckle" image. At right is shown the 15 second averaged $P S F$; these $P S F$ s are used in simulations of the curvature WFS to degrade images generated via Zemax so as to capture effects of atmospheric turbulence. For reference, a blue circle 0.6 " in diameter is overlaid. 
The simulation was advanced through 300 time steps of 50 milliseconds each via laterally shifting phase screens by an amount proportional to their velocity and re-propagating the wavefronts. Simulated 15 second time-averaged wavefront phases and point spread functions were formed by averaging over the 300 short exposure results (Figure 3). To accumulate adequate covariance statistics for the control system analysis, the entire simulation was repeated 100 times for the given $r_{0}$.

\subsection{Changes in gravity orientation}

The LSST Project has developed an Operations Simulator (OpSim) that models the time sequencing of observations given science priorities, the physical constraints of the design, and environmental constraints including weather and seeing. The scheduling algorithm used in OpSim penalizes visit priorities for large re-pointing distances; hence, the vast majority of visit re-pointing is to an adjacent field. The impact on AOS state variables from the rapid cadence is measured by the change in the projection of the gravity vector on the telescope structure from visit-to-visit. The change in the gravity vector projection along the optical axis is less than $10 \%$ for more than $95 \%$ of the time from one visit to the next and less than $3 \%$ in at least $50 \%$ of the visit-to-visit re-positioning. The projection normal to the optical axis has even smaller changes from visit-to-visit.

The AOS is principally operated off a Look-Up Table (LUT). The LUT provides open loop, near optimum values for all actuator forces and hexapod positions. The LUT values vary principally with elevation angle, i.e. gravity orientation.

Although the LUT values are near optimum, as a result of non-repeatable/predicable effects, they are inadequate to reach the image quality requirements. While for any given zenith angle the actuator forces are optimized for achieving the optimal mirror surface shape, there is always a small residual (print-through) that cannot be further reduced by actuators. Furthermore, actuator repeatability errors also contribute to the residual perturbations after LUT corrections. These effects are indicated in Figure 4 for the M1M3 substrate.

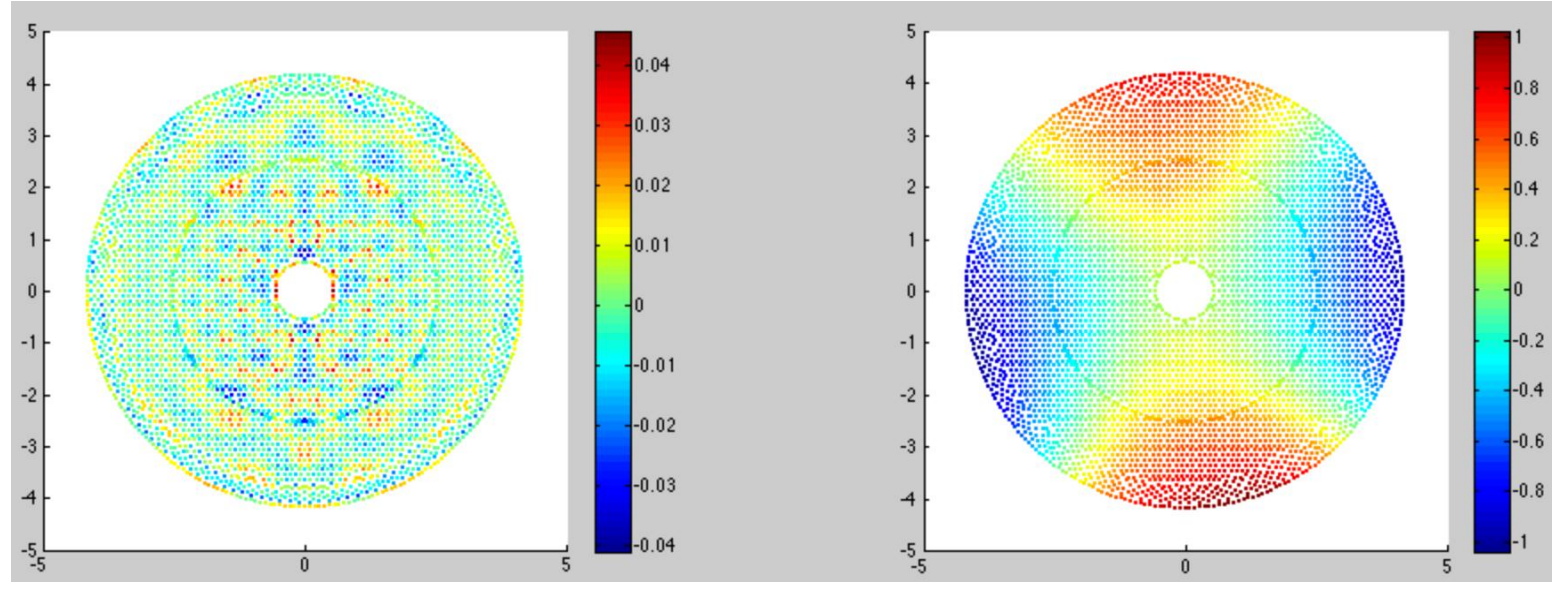

Figure 4 At left is shown the optimized surface (print-through in $\mu \mathrm{m}$ ) of the M1M3 substrate after LUT corrections of the gravitational deformations at $46^{\circ}$ elevation angle. At right is shown the same surface in $\mu \mathrm{m}$, after actuator repeatability errors (a conservative 5\% "noise") are added. It is worth to mention that the uniformly distributed actuator noise mostly excites the lowest order surface mode resembling an astigmatism.

The optical components in the camera are shifting with changing orientation of gravity due to deformations of the camera body and optical mounts. The gravity orientation in the camera depends on the telescope zenith angle, as well as the rotation of the camera following the virtual rotation of the sky. The rigid body displacements of L1, L2, filter and L3 are typically a few tens of microns, but can be up to $\sim 300 \mu \mathrm{m}$ depending on the zenith angle and camera rotation

The relative rigid body positions of M1M3, M2, and the camera are also changing with the direction of gravity. However, we assume we can build a LUT that can align these elements at least with the accuracy of the laser tracking system. 


\subsection{Temperature variations}

The other dominant state variable affecting the AOS is bulk temperature and its rate of change. The range of temperatures on Cerro Pachón spans a minimum of $-10^{\circ} \mathrm{C}$ up to a maximum of $30^{\circ} \mathrm{C}$. During the night it seldom gets above $16^{\circ} \mathrm{C}$ or below $2^{\circ} \mathrm{C}$. The mean rate of change at the beginning of the night is about $-1^{\circ} \mathrm{C} /$ hour.

The LSST utilizes a highly ventilated dome design to minimize the temperature difference between the inside and outside. The dome also incorporates a high capacity HVAC system operated during the daytime to set the internal dome temperature to $1^{\circ} \mathrm{C}$ below the expected start of night temperature. The $\mathrm{M} 1 \mathrm{M} 3$ thermal control system has been designed to accommodate at least a $1^{\circ} \mathrm{C} /$ hour temperature rate of change [7]. We are expecting the M1M3 thermal control system to control the thermal gradients in the M1M3 mirror substrate to within $0.1^{\circ} \mathrm{C}$, while it is following the ambient air temperature to $0.8^{\circ} \mathrm{C}$.

Figure 5 shows the evolution of ambient temperature during the day on Cerro Pachón. It also indicates the deformation of M1M3 substrate due to bulk temperature changes.
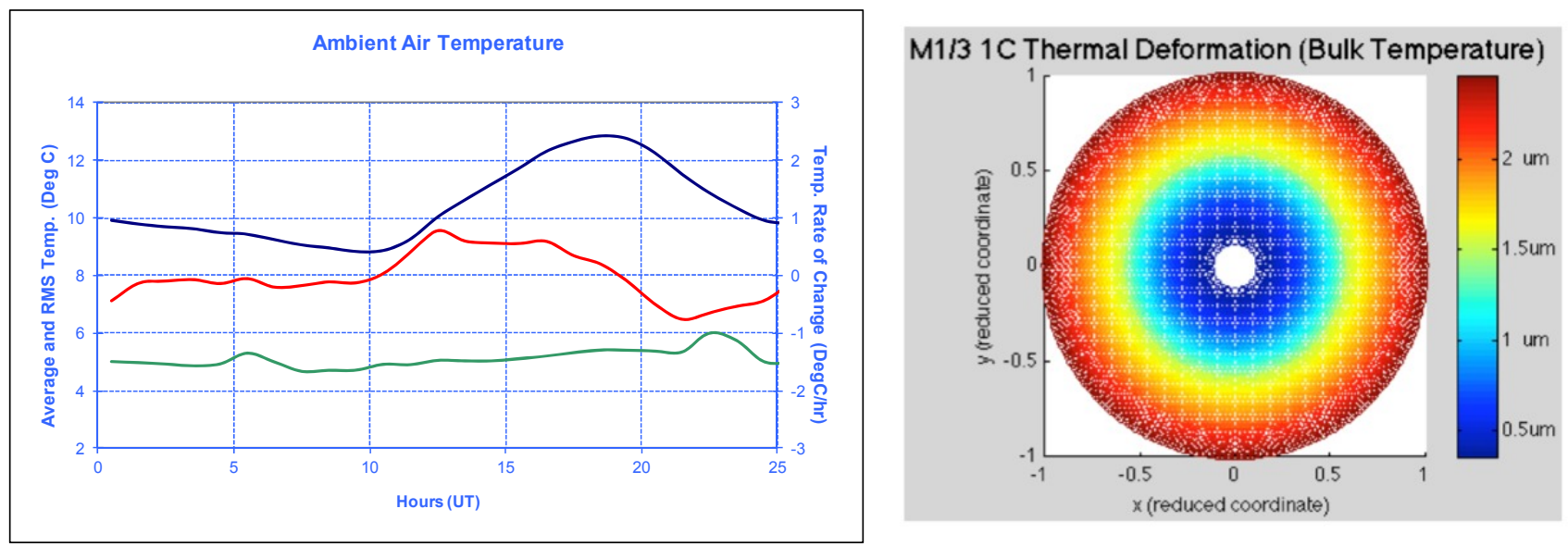

Figure 5 At left is shown the mean temperature on Cerro Pachón (blue, top curve), its standard deviation (green, lower most curve), and its rate of change (red, middle curve). At the right is shown M1M3 substrate deformation due to $1^{\circ} \mathrm{C}$ bulk temperature change.

Bulk temperature changes of the camera body result in expansions or contractions, which in turn affect the relative positions of the optical components in the camera. The camera body is temperature stabilized to the ambient (dome) air temperature with $1^{\circ} \mathrm{C}$ accuracy. Furthermore, due to adequate flushing facilitated by proper dome design, the internal (dome) air temperature is expected to follow external summit temperatures (Figure 5) fairly well.

Temperature changes will also affect the relative positions of the three major optical assemblies, M1M3, M2, and the camera. While eventually we expect to build a higher fidelity LUT to control these relative positions, for this study we assume the optical assemblies are aligned with the accuracy of the laser tracker system.

\subsection{Initial alignment}

Initial optical alignment of the LSST begins with extensive metrology and calibration of the optical control systems at the vendors prior to integration into the telescope. This metrology knowledge is then transferred to the as-built telescope and followed by in-situ optical testing.

The LSST telescope incorporates an on-board laser tracker. The laser tracker is located in the central hole of the M1M3 and secured to its mirror cell [1]. The laser tracker uses spherical mounted retro reflectors (SMRs) attached to the three optical assemblies to determine their relative orientation. The laser tracker system is only utilized when the camera is not operating. It will be used to preposition the optical assemblies before each night's observing and during integration to refine the Look-Up Table (LUT). The accuracy of determining the optical location of the 3 major assemblies by the laser tracker is summarized in Table 2 [1]. 
For the purpose of evaluating the performance of the AOS we assume as input perturbance the initial alignment accuracy of the telescope defined by the laser tracker system. It is actually a conservative estimate, as additional optical measurements with a Shack-Hartmann WFS, as well as the Commissioning Camera are planned before installing the full Camera, in order to refine the active optics LUT.

A similar laser positioning system is envisioned for the initial alignment of the camera optics, with expected accuracy summarized in Table 1.

Table 1 Expected accuracy of the camera alignment system

$\begin{array}{lcc}\begin{array}{l}\text { Optical } \\ \text { element }\end{array} & \begin{array}{c}\text { Degree of } \\ \text { Freedom }\end{array} & \text { Accuracy } \\ \begin{array}{l}\text { L1-L2 } \\ \text { assembly }\end{array} & \text { Piston } & 53 \mu \mathrm{m} \\ & \begin{array}{c}\text { Decenter } \\ \text { Tilt }\end{array} & 90 \mu \mathrm{m} \\ \text { Filter } & \text { Piston } & 161 \mu \mathrm{m} \\ \text { assembly } & \text { Decenter } & 213 \mu \mathrm{m} \\ & \text { Tilt } & 303 \mu \mathrm{rad} \\ \text { L3 assembly } & \text { Piston } & 47 \mu \mathrm{m} \\ & \text { Decenter } & 0(\text { reference }) \\ & \text { Tilt } & 143 \mu \mathrm{rad}\end{array}$

Table 2 Accuracy of the alignment of the 3 major optical assemblies by the laser tracker

\section{WAVEFRONT CURVATURE SENSORS (WCS)}

\subsection{Wavefront sensor architecture}

The LSST design will use 4 wavefront curvature sensors distributed at the corner of the inscribed square over the 3.5degree field of view (Figure 6). Curvature wavefront sensing offers some advantages over other wavefront sensing methods for wide field survey telescopes. By relying on equally defocused intra- and extra-focal images, curvature sensing can use area sensors with relatively large fields-of-view. This allows significant flexibility in selecting reference sources to use for the wavefront measurement when the source scenery is changing with the LSST visit cadence. Each LSST wavefront sensor uses a pair of sensors with $+/-1 \mathrm{~mm}$ focus offset to obtain the needed intra- and extra-focal images.
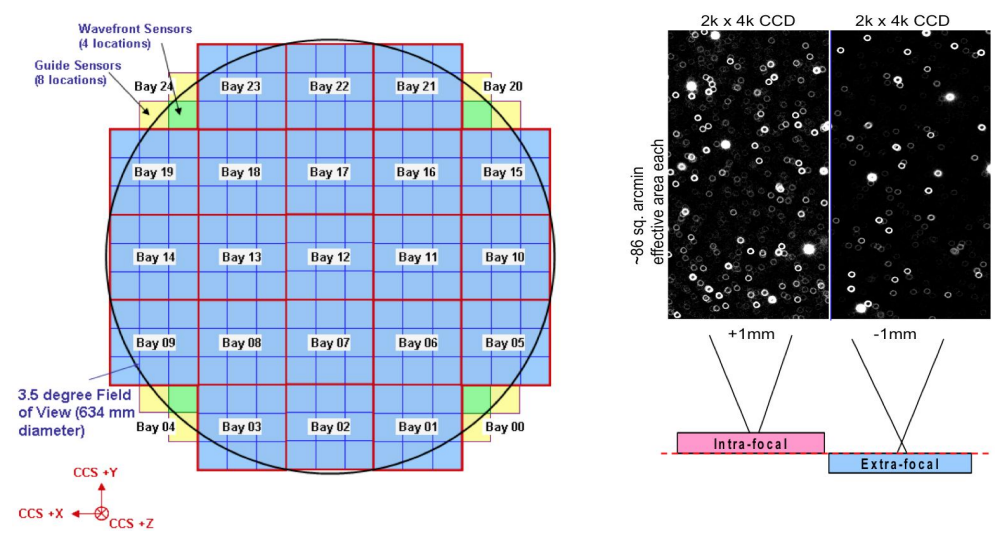

Figure 6 The geometry of LSST wavefront sensing. The focal plane configuration (left panel) showing sensors used for science (blue), guiding (yellow) and wavefront sensing (green). The wavefront sensors are divided into two halves of intra and extra focal sensors (right panel). 
Each WCS provides the first 22 Zernike coefficients to characterize the OPD at the exit pupil for the corresponding field position. Note that the first 3 coefficients (tip/tilt/piston) are not processed, as they do not have information about the shape of the wavefront. Guiding is facilitated by centroid measurements on different CCD segments.

\subsection{Algorithm modifications}

LSST's optical system poses algorithmic challenges using curvature sensing and solving the intensity transport equation for estimating the wavefront error at the entrance pupil:

(i) The highly annular pupil geometry means standard Zernike polynomials are not orthogonal; the Zernike terms we use to describe the exit pupil OPD (wavefront) are based on the annular polynomials.

(ii) The fast $\mathrm{f} / 1.23$ optical beam generates significant distortion if the system is projected to a plane; our algorithm removes this non-linear fast-beam distortion.

(iii) At the location of the wavefront sensors there are noticeable off-axis distortion and vignetting resulting in nonaxisymmetric intra- and extra-focal images; the LSST algorithm uses a polynomial mapping to correct for the distortion.

(iv) Field dependent variations in the wavefront over the wavefront sensor area must also be accounted for, as the intra- and extra-focal images do not belong to the same star.

\subsection{Algorithm validation}

The LSST curvature wavefront sensing algorithm was extensively tested and validated. Without the aspiration of being comprehensive, a few examples of algorithm linearity tests are shown in Figure 7. The wavefront sensor is exceptionally linear in the range of interest, even with the chosen $\pm 1 \mathrm{~mm}$ image separation; please note the large range of input mirror deformations given in waves.
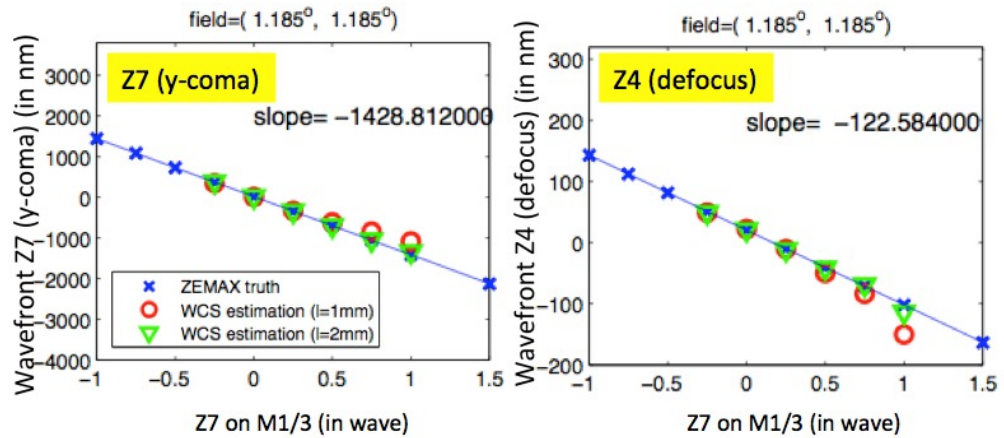

Figure 7 Representative examples of the WCS linearity tests validating functionality and performance.

The covariance of atmospheric Zernike coefficients was computed for 15-second time integrated Arroyo atmospheres, using the layer weighting described previously. The information is correlated across all four wavefront sensors due to the ground layer turbulence.
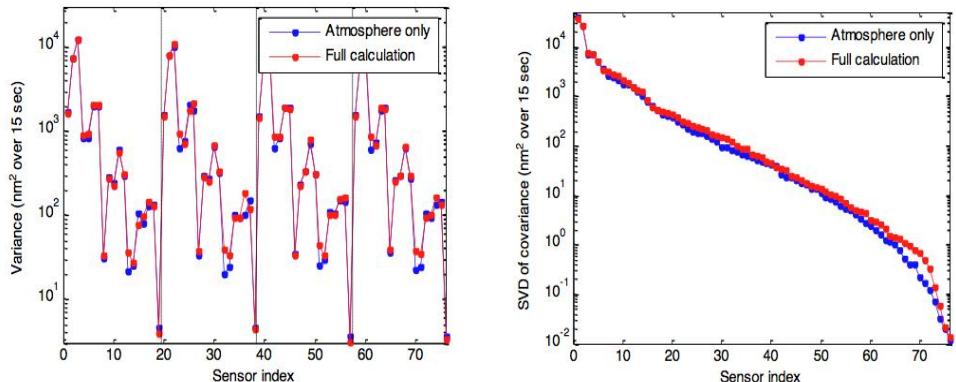

Figure 8 The nearly identical diagonal elements (left) and singular values (right) of the covariance matrices for the ideal sensor (atmosphere only) and the fully implemented WCS using simulated images indicate negligible algorithmic noise, compared to the atmospheric effects. 
The same time-integrated phase screens used for the atmosphere covariance were applied to a Zemax ray-trace model in order to evaluate the importance of algorithmic noise with respect to atmospheric noise in estimating the wavefront. For each time-integrated phase screen instance, Zemax was used to generate intra- and extra-focal image pairs at each of the 4 wavefront sensors. These images were processed through the LSST curvature algorithm to estimate the wavefront coefficients. When compared to the derived coefficients from atmosphere alone (ideal sensor), the actual sensor algorithm results are very similar, indicating that the covariance is dominated by the atmospheric contribution and not the wavefront sensing algorithms. The plots in Figure 8 show the diagonal entries of the covariance (19 entries for each field point) and the singular values of the covariance matrix; the largest singular values most important for estimating displacements are practically identical.

\section{OPTICAL FEEDBACK CONTROL ARCHITECTURE AND DESIGN}

\subsection{Control model}

The model used to design and simulate the optical (wavefront) feedback loop for the LSST AOS is shown in Figure 9. The Plant to be controlled includes the (i) optical components and their mounting structures both in the telescope and camera, and the (ii) optical system forming images on the focal surface. At this point it does not include the effects inside the silicon (CCD). The outputs of the system are the (i) archieved image quality [q] measured in FWHM and the (ii) exit pupil wavefront (OPD) estimated by the WCS in terms of Zernike coefficients [y] for the feedback loop.

The overall feedback algorithm can explicitly be separated into two steps: (i) estimating the perturbations (system states, $\hat{\mathbf{x}}$ ) from the wavefront, and (ii) choosing a control response [u] given the estimate. Because of the separation principle, the optimal estimate does not depend on what the control does with it, and the optimal control is the same as what it would be if the state estimate was perfect. The estimator can be thought of as a "spatial" reconstructor, while the controller defines the (temporal) dynamic behavior.

Table 3 Controlled degrees of freedom $[\mathbf{x}]$ in AOS

$\begin{array}{lll}\text { Optical element } & \begin{array}{l}\text { Degrees of Freedom } \\ \text { M2 }\end{array} & \begin{array}{l}\text { Piston } \\ \text { x/y decenter } \\ \text { x/y tilt }\end{array} \\ \text { Camera as a whole } & \begin{array}{l}\text { Piston } \\ \text { x/y decenter } \\ \text { x/y tilt }\end{array} & \text { Realized by the M2 hexapod } \\ \text { M1M3 substrate } & \begin{array}{l}\text { Shape } \\ \text { (\#1-\#15 bending modes by the Camera hexapod/rotator } \\ \text { of the M1M3 substrate) } \\ \text { Shape } \\ \text { (\#1-\#15 bending modes) }\end{array} & \begin{array}{l}\text { The full set of (156-3) bending modes form a full basis set for all } \\ \text { possible shapes the 156 figure control actuators can generate. }\end{array} \\ & \begin{array}{l}\text { The full set of (72-3) bending modes form a full basis set for all } \\ \text { possible shapes the 72 figure control actuators can generate. }\end{array}\end{array}$

The controlled, actual system states $[\mathbf{x}]$ define the optical response, together with the uncontrolled perturbations $\left[\mathbf{x}_{2}\right]$. Atmospheric effects are added to the wavefront measurements as a Zernike coefficient vector [w]. In order to clearly understand the image quality degradation of the system itself and be able to compare it to the requirements captured in the error budget, atmospheric effects are not included in the FWHM output. The controlled degrees of freedom are summarized in Table 3.

The wavefront control loop was optimized and initially evaluated with a linear model:

(i) A sensitivity matrix (A, pictured in Figure 10) links the Zernike coefficients of the exit pupil OPD (y) to the various controlled degrees of freedom of the system; a similar sensitivity matrix $\left(\mathbf{A}_{\mathbf{2}}\right)$ links the uncontrolled perturbations $\left(\mathbf{x}_{\mathbf{2}}\right)$ to the exit pupil OPD. The sensitivity matrix is the small signal Jacobian of the Zernike expansion with respect to all controlled (or uncontrolled) degrees of freedom. 
(ii) Atmospheric effects and WCS noise are represented by the random vector variable $\mathbf{w}$ derived from the Arroyo model; and

(iii) The image quality metric (weighted rms $F W H M$ across the field) is expressed as the rms value of a variable (q), which in turn relates to the Zernike expansion of the pupil OPD (y) (see section 4.2).

$$
\begin{aligned}
\mathbf{y} & =\mathbf{A x}+\mathbf{A}_{2} \mathbf{x}_{\mathbf{2}}+\mathbf{w} \\
\mathbf{q}^{2} & =\mathbf{y}^{\mathrm{T}} \mathbf{Q} \mathbf{y}
\end{aligned}
$$

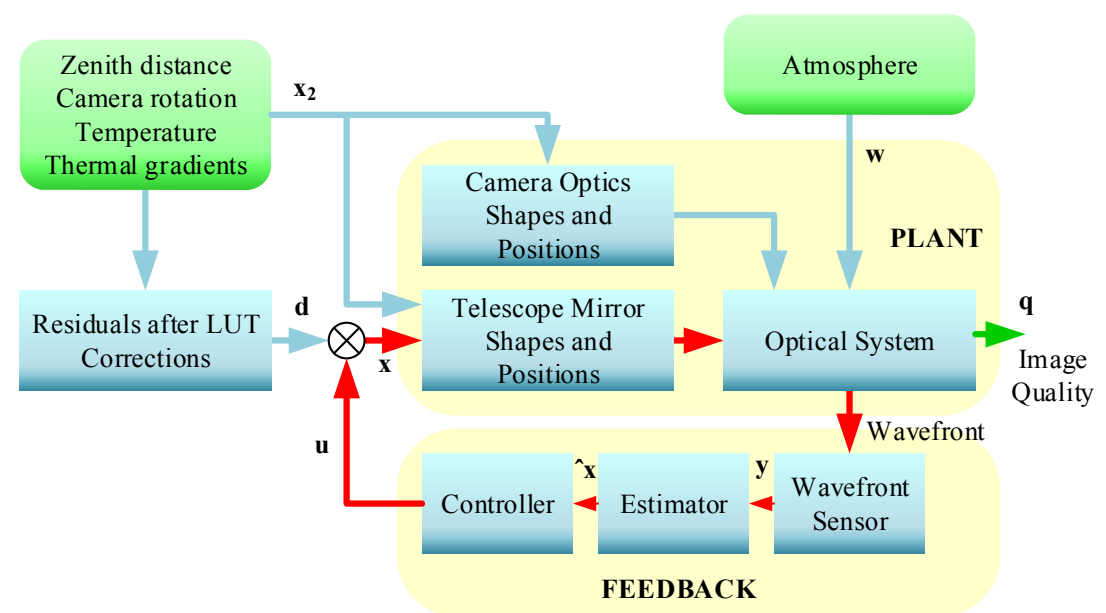

Figure 9 The wavefront control model of the LSST AOS (see text for the explanation of signals shown)

The near degeneracy of the sensitivity matrix is clearly visible in Figure 10. Some output variables are hardly influenced by any of the input degrees of freedom; in particular, Z11 (spherical), Z16, Z17, and Z22 are obviously barely controllable. (The Zernike terms are shifted up in Figure 10, as the first 3 terms (piston/tip/tilt) are omitted.) As a result, if any spherical aberration exists in the measurements - due to atmospheric effects, for example, - a naïve feedback loop will command huge changes in the controlled degrees of freedom in order to compensate. Our feedback design mitigates this shortcoming with a truncated Estimator and optimal Controller, as described in subsequent sections.

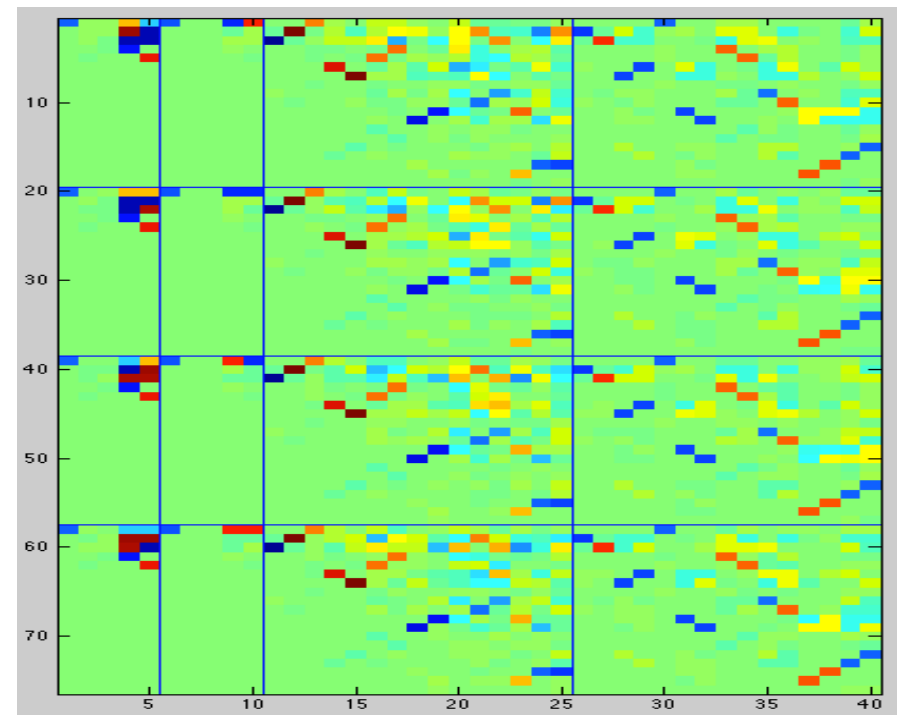

Figure 10 The linear sensitivity (influence) matrix A capturing the small signal partial derivatives of the measured wavefront Zernike coefficients to the various controlled degrees of freedom in the system. The horizontal blue gridlines are separating the measurements of the $4 \mathrm{WCS}$, while the vertical dividers indicate the four groups of those controlled degrees of freedom: M2 rigid body positions, Camera rigid body positions, M1M3 bending modes, and M2 bending modes. Red indicates positive, blue negative values; green corresponds to zero. 


\subsection{Performance metric}

LSST being a very large Field-of-View telescope, the image quality may vary across the full field by non-negligible amounts. Consequently, the relevant performance metric is the mean FWHM over the focal plane, regularly estimated by the Gaussian Quadrature method. The distribution of sampling points used is shown in Figure 11 .

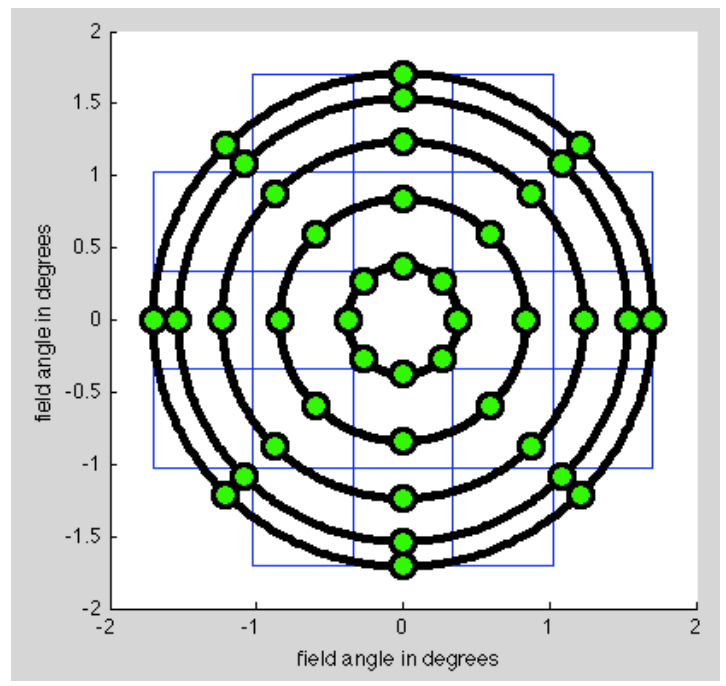

Figure 11 Image quality sampling point on the focal plane used to evaluate the mean FWHM
Let's assume there is a unique relationship between the $F W H M$ at a field position to the wavefront Zernikes at the same field position. If each Zernike term affected the FWHM in independent ways, their relation should be largely quadratic. Considering that the shift in the ray hit position on the image plane is proportional to the slope of the wavefront at the pupil, an empirical approximation can be devised.

$$
\begin{aligned}
\text { FWHM }_{f}^{2} & \approx \sum_{i}\left(a_{f, i} y_{i}^{2}+b_{f, i} y_{i}\right)+ \\
& +\sum_{i, j}\left(\operatorname{cor}_{f, i, j} y_{i} y_{j}\right)+c_{f}^{2}
\end{aligned}
$$

Here $f$ is the field number, $y_{i}$ is the rms of the $i^{\text {th }}$ order annular Zernike without LSST design aberration, $a, b$, and $c$ are constants to be fitted, and cor denotes the cross-term correlation coefficients.

Numerical fitting leads to the conclusion that the linear term $b$ is generally small and the field-dependent constant $c$ well approximates the image quality of the unperturbed LSST in terms of FWHM. The dominantly quadratic relationship can be described by a matrix $\mathbf{Q}$. Note that the matrix also includes the weights of the Gaussian Quadrature method.

$$
\mathbf{q}^{2} \approx \mathbf{y}^{T} \mathbf{Q} \mathbf{y}+\mathbf{c}^{2}
$$

\subsection{Estimator}

We have a set of wavefront measurements $y$ with which we want to estimate (and then control) the telescope state $\mathbf{x}$ The measurements $y$ have sensor noise $w$ with (fully-populated) covariance matrix $\mathbf{W}$ due to both algorithmic and atmospheric contributions. Furthermore, $y$ responds to perturbations $\mathbf{x}_{2}$ that are not intended to be controlled but will in general be nonzero. To make a minimum-variance estimate of $x$ in the presence of non-zero noise $\mathbf{w}$, we also need to know how "large" to expect $\mathbf{x}$ to be, which can be quantified by the covariance matrix $\mathbf{X}$.

Note that $\mathbf{A}_{2} \boldsymbol{x}_{2}$ can introduce aliasing wherein an actual motion within some component of $\mathbf{x}_{2}$ results in a nonzero value of $y$ and an erroneous estimate of $\mathbf{x}$. This is likely to occur, for example, if higher-order Zernikes are included in the wavefront sensors than those corrected on M2 and M1M3. Combining both "errors" in the measurement vector $\mathbf{y}$ above, we can create a covariance matrix $\mathbf{W}_{2}=\mathbf{W}+\mathbf{A}_{2} \mathbf{X}_{22} \mathbf{A}_{2}{ }^{\mathrm{T}}$.

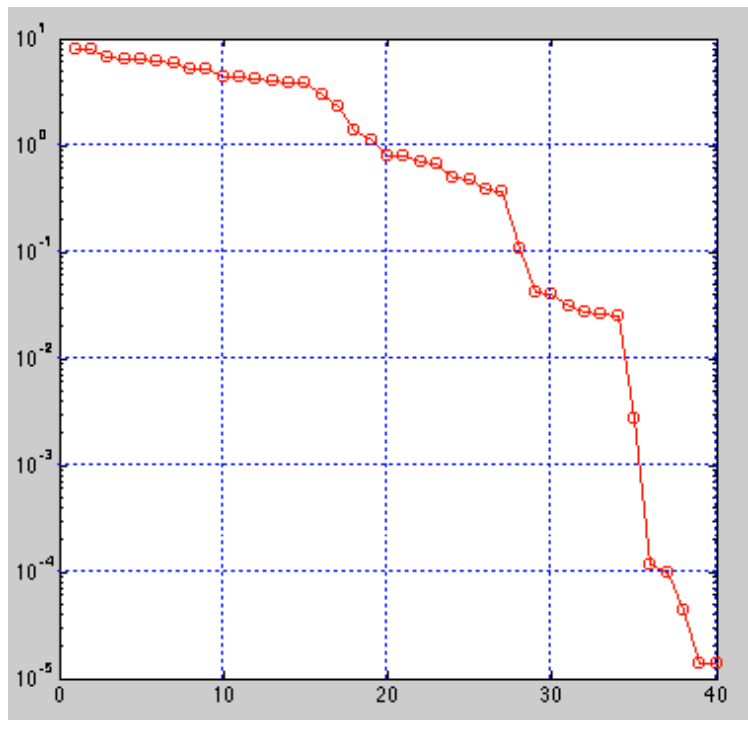

Figure 12 Singular values of the influence function A shown in Figure 10 
In principle the optimal estimate including this full covariance matrix should avoid aliasing by avoiding reliance on sensor information that is uncertain due to the un-estimated degrees of freedom $\mathbf{x}_{\mathbf{2}}$. In practice, $\mathbf{A}_{\mathbf{2}} \mathbf{x}_{\mathbf{2}}$ has a small effect on the estimates and is thus neglected here.

The minimum variance estimate of $\mathbf{x}$ is independent of the sensor and state dimensions $m$ and $n$, though clearly there will be a poor estimate of $\mathbf{x}$ if $m<n$.

$$
\hat{\mathbf{x}}=\mathbf{X} \mathbf{A}^{T}\left(\mathbf{A X} \mathbf{A}^{T}+\mathbf{W}\right)^{-1} \mathbf{y}
$$

However, the argument can be made that both $\mathbf{X}$, characterizing the perturbations, and $\mathbf{W}$, mostly depending on the strength of atmospheric turbulence are varying and somewhat unpredictable parameters. Consequently, full optimization of the estimator is a fairly involved procedure. For this study, we use the pseudo-inverse of the influence matrix, which is clearly Equation (3.4) at the limit of $\mathbf{W}=0$.

$$
\hat{\mathbf{x}}=\mathbf{A}^{T}\left(\mathbf{A} \mathbf{A}^{T}\right)^{-1} \mathbf{y}
$$

The sensitivity matrix $\mathbf{A}$ is full rank, and therefore in the absence of noise, the wavefront sensors provide sufficient information to estimate all of the perturbances x. However, in the presence of noise, there are "directions" that are practically degenerate. This is best characterized by looking at the singular values of A (Figure 12). The very small singular values at the "tail" of the graph indicate linear combinations of system degrees of freedoms (singular modes) that have practically no influence on the measurement. On the other hand, after inverting the A matrix, these very singular values become huge and can lead to significant noise propagation into the barely observable singular modes ("virtual" perturbations).

These poorly observed displacement patterns also have relatively small impact on the optical performance, though. In other words, what we cannot measure in the optical response of the system has negligible impact on system performance. Consequently, the 5 smallest singular values of $\mathbf{A}$ can be neglected in the matrix inversion; the rest of the matrix provides adequate wavefront information to estimate the displacements with sufficient accuracy.

Note that some noise accumulation in these barely visible degrees of freedom may happen, anyway. To avoid uncontrolled large drifts over time, these modes also need to be adjusted based on wavefront information accumulated during longer time. The longer integration time assures small enough noise levels for the system to take advantage of the full rank of the A matrix by using all the singular values.

\subsection{Optimal controller}

For simplicity, we decided to use an integral controller in our study. Its gain, $|\alpha| \leq 1$ determines the speed of convergence through iterations $(k)$.

$$
\mathbf{x}_{k+1}=\mathbf{x}_{k}+\alpha \mathbf{F} \hat{\mathbf{x}}_{k+1}+\mathbf{d}_{k+1}
$$

The coefficient matrix $\mathbf{F}$ here can be designed to minimize a particular cost function $J$. In our case, optimizing image quality, i.e. minimizing the variance of $F W H M$ is an obvious goal. Furthermore, we want to limit large actuator swings to avoid damage to the glass, as well as to ensure smooth transitions between iterations. This additional goal can be achieved by incorporating the variance of the actuator commands (u) in the cost function with some weight $\rho$. The matrix $\mathbf{H}$ defines the distribution of control authority among various actuator groups (rigid body and shape actuators) such that $1 \mu \mathrm{m}$ or 1 arcsec rigid body displacement corresponds to $1 \mathrm{~N}$ actuator force.

$$
J=\mathbf{y}^{T} \mathbf{Q} \mathbf{y}+\rho \mathbf{u}^{T} \mathbf{H} \mathbf{u}
$$

For the current study, the weight was set in favor of the $F W H M$ goal: the cost of 0.1 mas $F W H M$ error was equivalent to that of $1 \mathrm{~N}$ actuator force. Both $\rho$ and $\mathbf{H}$ can be further optimized in future studies.

Substituting the measurement Equation (3.1) into the cost function and differentiating regarding to $\mathbf{u}$ yields the optimal $\mathbf{F}$ matrix for minimizing the given cost function.

$$
\mathbf{F}=\left(\mathbf{A}^{T} \mathbf{Q A}+\rho \mathbf{H}\right)^{-1} \mathbf{A}^{T} \mathbf{Q A}
$$


By combining the Estimator and Controller, we can define the feedback algorithm.

$$
\mathbf{x}_{k+1}=\mathbf{x}_{k}+\alpha\left(\mathbf{A}^{T} \mathbf{Q} \mathbf{A}+\rho \mathbf{H}\right)^{-1} \mathbf{A}^{T} \mathbf{Q} \mathbf{y}_{k+1}+\mathbf{d}_{k+1}
$$

\section{SIMULATION RESULTS}

\subsection{Linear model}

We verified that the derived control feedback is properly working in the linear environment it was devised in. Setting the integral gain to 0.3 results in longer integration time, which in turn leads to better noise suppression (Figure 13). Similar settings can be useful for fine tuning the LUT, as well as - in a parallel loop - recovering barely observable modes to prevent long term drift of those modes.

In regular operations, convergence speed is critical. While it is acceptable to occasionally lose an observation due to outof-spec image quality, especially after longer slews, it is preferable to recover in a single iteration and not to lose further observations. As Figure 14 demonstrates, it is feasible by setting the gain to 1 . The disadvantage of this setting is poorer noise suppression, resulting in somewhat larger command swings.

Since the linear model is using the sensitivity matrix to represent the optical system, all the effects included are projected on the domain and range of this transformation. All the mirror shapes are truncated to the controlled bending modes and all the noise and uncontrolled perturbations are expressed in the observed Zernike terms. Furthermore, image quality is not directly observable - there is no image in this model, - but rather it is estimated from the Zernike expansion of the exit pupil. The lack of image also means that the WCS algorithm could not be used; wavefront "measurements" were equal to the exit pupil OPD with added atmospheric phase noise.
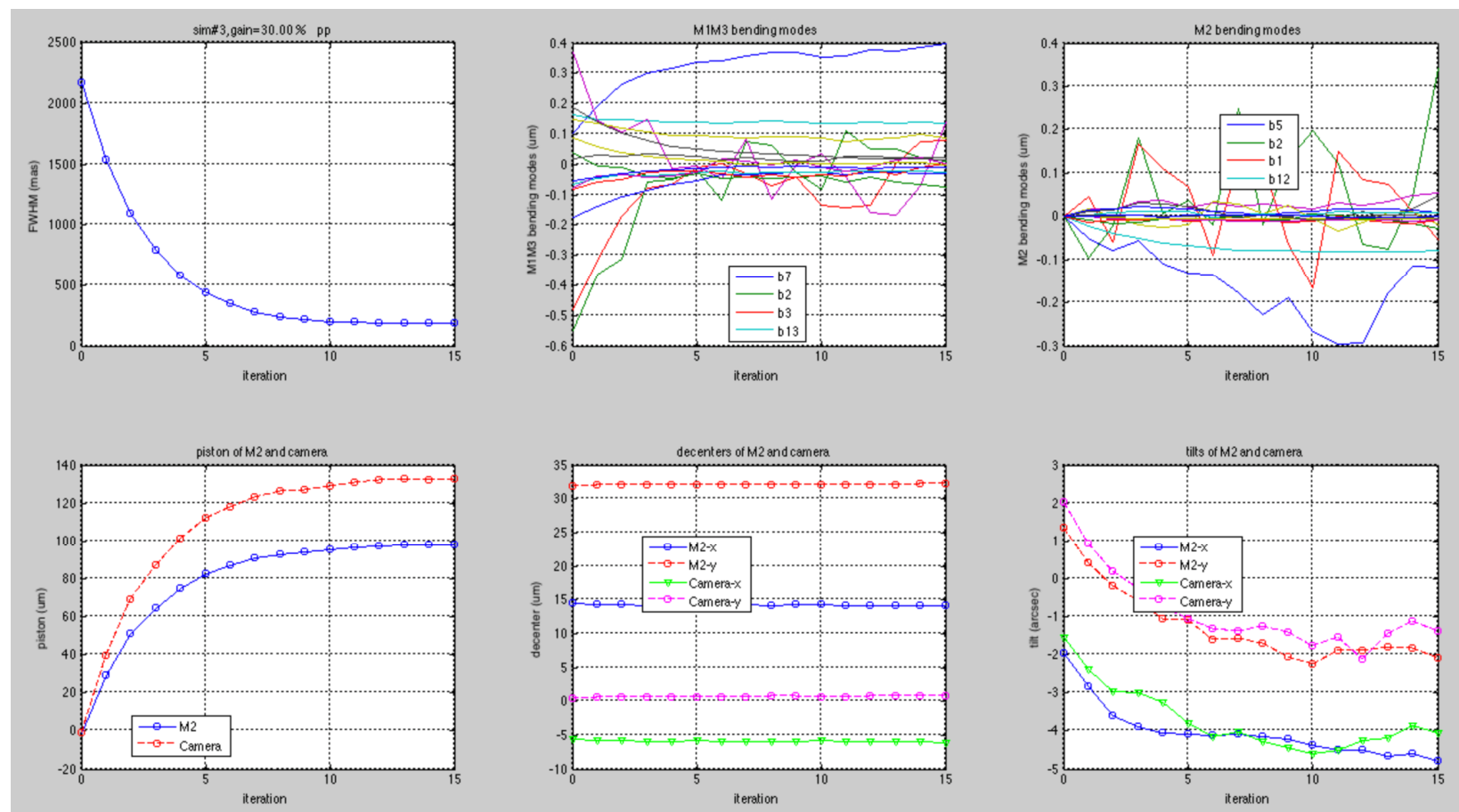

Figure 13 Linear system response with $\alpha=0.3$, showing convergence of $F W H M$ through several iterations (sampling) in the upper left graph. The other graphs indicate the evolution of various controlled system degrees of freedom. Please note the simulated FWHM is slightly below the error budget requirement of 217 mas for the errors included in the simulations. 

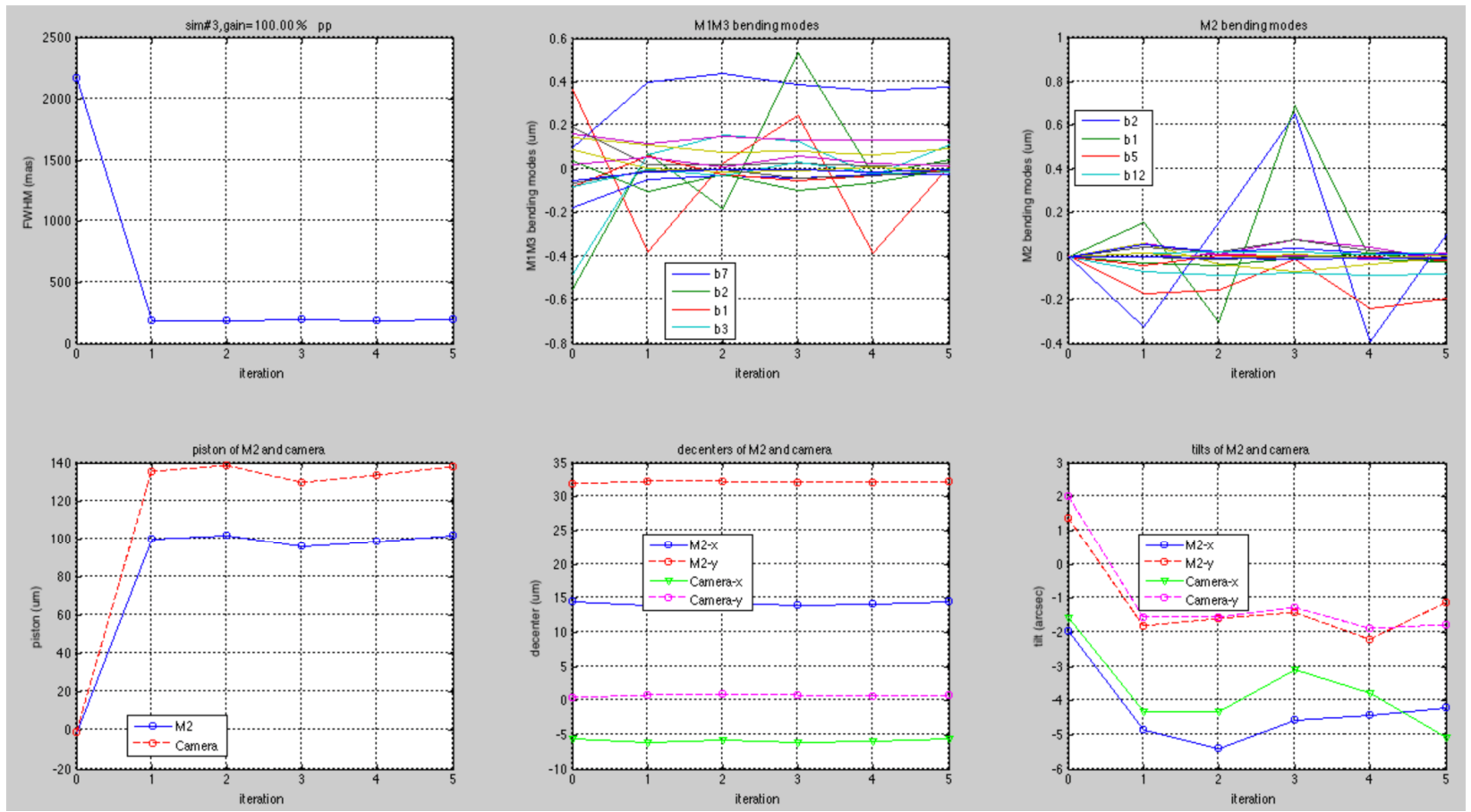

Figure 14 Linear system response with $\alpha=1$, showing convergence of $F W H M$ in a single iteration (upper left graph). The other graphs indicate the evolution of various controlled system degrees of freedom. Please note the simulated $F W H M$ is slightly below the error budget requirement of 217 mas for the errors included in the simulations.

\subsection{Nonlinear model}

To validate the results of the linear approximation used to design and evaluate the optical feedback system, we carried out nonlinear simulations that are more representative of the real system:

(i) Zemax was directly linked to matlab to replace the optical sensitivity matrix with actual ray tracing. This is the same Zemax model that was used for generating the sensitivity matrix.

(ii) The actual LSST WCS algorithm was applied to intra- and extra-focal images simulated by Zemax in the presence of atmospheric aberrations.

(iii) The $F W H M$ was determined from simulated $P S F$ s to replace the linear approximation linking $F W H M$ to exit pupil wavefront Zernike expansion.

The results shown in Figure 15 are remarkably similar to the linear simulation results in Figure 14. 

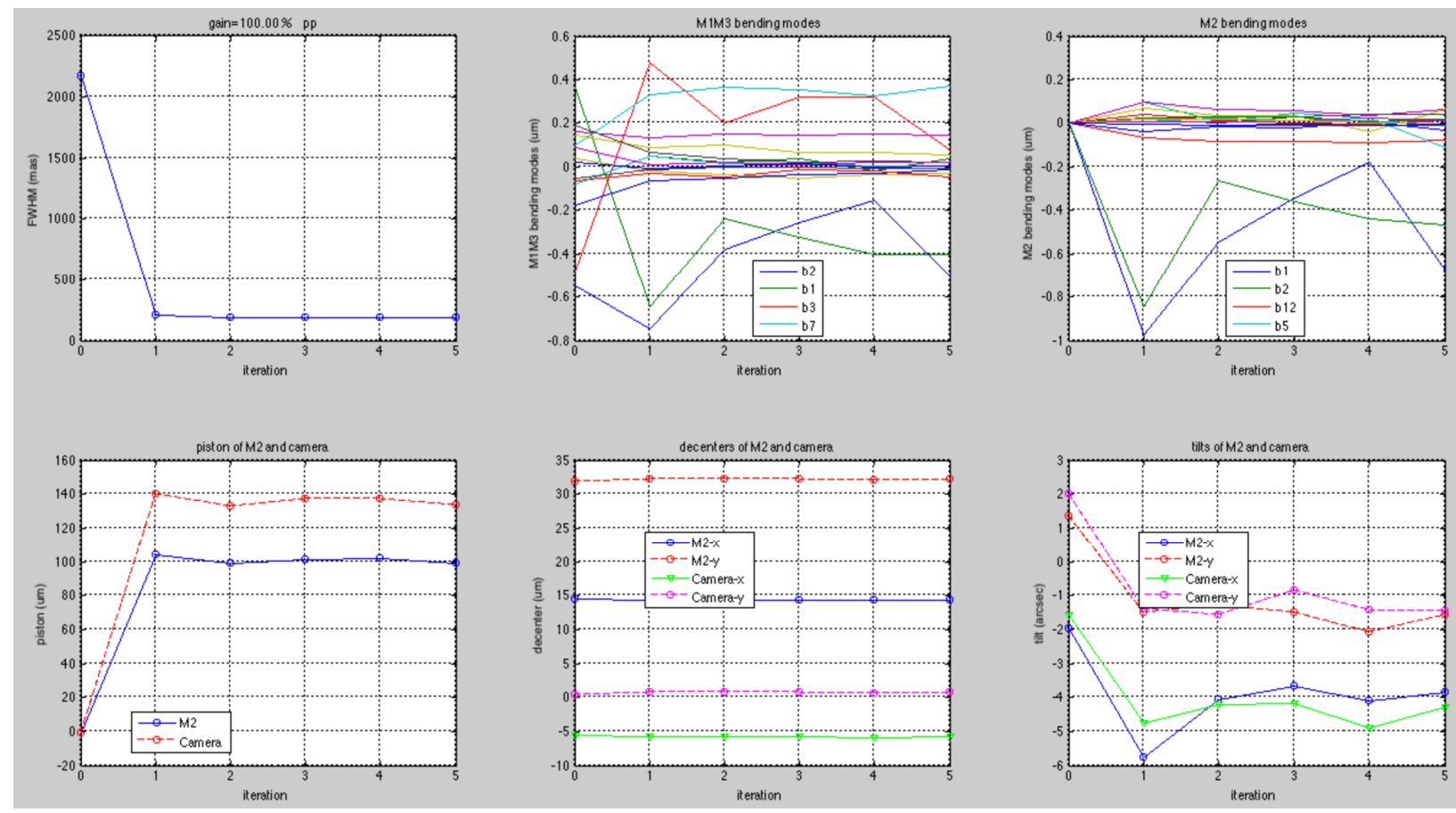

Figure 15 Nonlinear system response with $\alpha=1$, showing convergence of $F W H M$ in a single iteration (upper left graph). Note the similarity of system response to the corresponding linear results in Figure 14. The other graphs indicate the evolution of various controlled system degrees of freedom. Please note the simulated FWHM is slightly below the error budget requirement of 217 mas for the errors included in the simulations.

\section{CONCLUSION}

We demonstrated that the near degeneracy of the three mirror optical system of the LSST can be mitigated by a welldesigned wavefront control loop. Such a loop neglects the barely observable system states in its immediate response, corresponding to the principle that immeasurable optical response means negligible image degradation. However, a longer integration time second loop is envisioned - but not simulated yet - to limit long term drift in these hardly observable system modes.

Furthermore the usefulness of an optimal control law was confirmed for preventing large command swings, while maintaining proper convergence and control efficiency.

Future work is required to further optimize control performance and improve the fidelity of the system model utilized.

- More rigorous observability/controllability analysis can guide the design in deciding which bending modes are needed for more efficient control and which Zernike terms are worth measuring.

- Further exploring cost function weights can lead to a more balanced and efficient distribution of control authority among the actuator groups.

- Gaining more information about the evolution of perturbations to the system can result in an Estimator optimized for the particular perturbations

- Focal plane perturbations need to be included, in particular the geometric shape and position of the CCDs.

- The overall sensitivity matrix used in the feedback loop can only be measured on the summit during commissioning, even if various components, such as the mirror shape influence functions can be calibrated in the laboratory. Consequently, the performance consequences of potential uncertainties in the matrix need to be understood. 
- To enable weak lensing measurements, the image ellipticity of LSST is tightly controlled. The simulation framework reported is capable of predicting ellipticity, besides image size, with proper post-processing to be implemented.

\section{ACKNOWLEDGMENTS}

LSST project activities are supported in part by the National Science Foundation through Cooperative Support Agreement (CSA) Award No. AST-1227061 under Governing Cooperative Agreement 1258333 managed by the Association of Universities for Research in Astronomy (AURA), and the Department of Energy under contract with the SLAC National Accelerator Laboratory. Additional LSST funding comes from private donations, grants to universities, and in-kind support from LSSTC Institutional Members.

\section{REFERENCES}

[1] Neill, D., Angeli, G., Claver, C., Hileman, E., DeVries, J., Sebag, J., Xin, B., "Overview of the LSST Active Optics System," Proc. SPIE 9150, (2014)

[2] Phillion, D.W., Olivier, S.S., Baker, K., Seppala, L., Hvisc S., "Tomographic wavefront correction for the LSST," Proc. SPIE 6272, (2006)

[3] Manuel, A. M., Phillion, D.W., Olivier, S.S., Baker, K.L., Cannon B., "Curvature wavefront sensing performance evaluation for active optics correction of the Large Synoptic Survey Telescope (LSST)," Optics Express 18(2), (2010)

[4] Liang, M., Krabbendam, V., Claver, C.F., Chandrasekharan, S., Xin B.,"Active Optics in Large Synoptic Survey Telescope," Proc. SPIE 8444, (2012)

[5] Schechter, P.L., Levinson R.S., "Generic misalignment patterns in wide-field telescopes," PASP 123, (2011)

[6] Britton, M., "Arroyo," Proc. SPIE 5497, (2004)

[7] Neill, D., "Cooling Flow Requirements for the Honeycomb Cells of the LSST Cast Borosilicate PrimaryTertiary Mirror," Proc. SPIE 7424, (2009) 